\title{
Photon antibunching in the optical near field
}

\author{
R. Marty, A. Arbouet, V. Paillard, and C. Girard \\ CEMES, UPR 8011, CNRS-Université de Toulouse, 29 rue Jeanne Marvig, BP 94347, F-31055 Toulouse, France \\ G. Colas des Francs \\ LICB, UMR 5209, CNRS-Université de Bourgogne, 9 Avenue A. Savary, BP 47870, F-21078 Dijon, France
}

(Received 24 June 2010; published 9 August 2010)

\begin{abstract}
We show that a combination of the field-susceptibility technique with the optical Bloch equations gives access to the temporal evolution of the populations and coherences of any quantum system placed in the optical near field of a nanostructure. In particular, we show that the near-field evanescent states, confined around dielectric or plasmonic particles, can be used to modify and control the photon statistics of the quantum system. This theoretical scheme leads to second-order autocorrelation functions in good agreement with recent experimental measurements performed with nitrogen-vacancy center in diamond nanocrystals placed in interaction with gold nanoparticles.
\end{abstract}

DOI: 10.1103/PhysRevB.82.081403

PACS number(s): 78.20.Bh, 42.50.Ar, 07.79.Fc

In the last 20 years, a tremendous interest has been devoted to the study of the optical near field generated in the vicinity of dielectric or plasmonic nanostructures. ${ }^{1,2}$ Controlling the near field enables light transfer in highly reduced geometries and paves the way for future optical technologies, integrable, and scalable down to molecular dimensions. ${ }^{3,4}$ In a closely related context, single molecules or quantum dots are often used as efficient local sensors of the plasmonic near-field intensity. ${ }^{5-7}$ Very recently, the direct control of the photon statistics of various quantum systems (QSs) coupled with plasmonic structures has been demonstrated. $3,4,8-10$

In particular, recent measurements of the second-order photon autocorrelation function $g^{(2)}(\tau)$ are well illustrated into elegant experiments where local probe-based methods are used to tune the spacing between a single quantum system and a plasmonic nanostructure. ${ }^{9,10}$ For example, impressive photon antibunching control has been recently reported ${ }^{9}$ by approaching gold nanoparticles close to single nitrogenvacancy $(\mathrm{NV})$ color center in diamond nanocrystals with the tip of an atomic force microscope.

In this Rapid Communication, we generalize the theoretical framework described in Ref. 11 to the study of the photon statistics of a quantum system coupled with complex nano-optical structures, including dielectric, metallic, or hybrid materials. The approach is based on the combination of the field-susceptibility/Green's-tensor technique with the optical Bloch equations. ${ }^{12}$ The former provides an accurate electromagnetic description of the system, including both the local electric field and local density of states. ${ }^{13,14}$ These two techniques are merged to describe time-dependent populations, correlation functions, and antibunching characteristic times. Specific applications on realistic systems ${ }^{9,10}$ are described in the second part of the Rapid Communication.

Let us consider a single QS located at the position $\mathbf{R}_{q s}=\left(X_{q s}, Y_{q s}, Z_{q s}\right)$ in the near field of a nanostructure and characterized by its dipolar operator $\hat{\mathbf{m}}$ and Hamiltonian $\hat{H}_{0}$ (Fig. 1). The whole system is illuminated by a monochromatic plane wave of electric field $\mathbf{E}_{0}$ and frequency $\omega_{0}$. The QS is treated as a two-level system $\{|g\rangle,|e\rangle\}$, where $\omega_{e g}$ is the frequency of the transition $(|g\rangle \rightarrow|e\rangle)$ and $\delta_{L}=\omega_{e g}-\omega_{0}$ is the detuning. The dipolar coupling Hamiltonian $\hat{W}(t)=-\hat{\mathbf{m}} \cdot \mathbf{E}\left(\mathbf{R}_{q s}, t\right)$ can then be deduced from the local field $\mathbf{E}\left(\mathbf{R}_{q s}, t\right)$,

$$
\hat{W}(t)=-\sum_{\alpha} \hat{m}_{\alpha} \mathcal{E}_{\alpha}\left(\mathbf{R}_{q s}, \omega_{0}\right) \cos \left[\omega_{0} t+\phi_{\alpha}\left(\mathbf{R}_{q s}\right)\right]
$$

where $\mathcal{E}_{\alpha}\left(\mathbf{R}_{q s}, \omega_{0}\right)$ and $\phi_{\alpha}\left(\mathbf{R}_{q s}\right)$ (with $\alpha=x, y$, or $z$ ) represent the magnitudes and the phases of the three Cartesian components of $\mathbf{E}\left(\mathbf{R}_{q s}, t\right)$. Applying the usual rotating-wave approximation, ${ }^{12}$ one obtains

$$
\hat{W}(t)=\sum_{\alpha=x, y, z} \frac{\hbar \Omega_{\alpha}\left(\mathbf{R}_{q s}\right)}{2}\left\{e^{-i\left(\omega_{0} t+\phi_{\alpha}\left(\mathbf{R}_{q s}\right)\right)}|e\rangle\langle g|+\text { c.c. }\right\},
$$

where $\Omega_{\alpha}\left(\mathbf{R}_{q s}\right)$ is the $\alpha$ component of the vectorial Rabi frequency. This quantity which characterizes the strength of the interaction between the incident electric field and the quantum system is proportional to the $\alpha$ component of the absorption transition dipole $m_{e g, \alpha}=\left\langle e\left|\hat{m}_{\alpha}\right| g\right\rangle$,

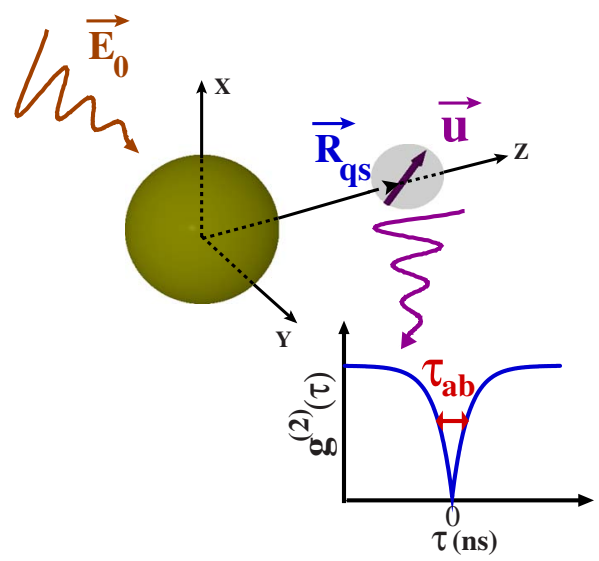

FIG. 1. (Color online) Schematic representation of a QS interacting with an arbitrary optical environment represented by a gold sphere. The unit vector $\mathbf{u}$ defines the orientation of the transition dipole moment. 


$$
\Omega_{\alpha}\left(\mathbf{R}_{q s}\right)=-\frac{m_{e g, \alpha} \mathcal{E}_{\alpha}\left(\mathbf{R}_{q s}, \omega_{0}\right)}{\hbar} .
$$

From the coupling Hamiltonian (2), we can calculate the time-dependent quantities (populations, coherences, and fluctuating dipole moment) to finally describe the photon statistics of the emitter. This can be achieved by numerically solving the time-dependent density matrix $\rho(t)$ evolution equation,

$$
\dot{\rho}(t)=\frac{1}{i \hbar}\left\{\left[H_{0}+W(t)\right], \rho(t)\right\}+\mathcal{R}_{\text {spont }} \rho(t)
$$

with a time-domain finite-difference method. The Redfield operator $\mathcal{R}_{\text {spont }}$ describes the coupling with the photon bath.

The photon emission of a single two-level system is antibunched because the generation of a photon projects the QS wave function into the ground state from which a second photon cannot be emitted. This pure quantum phenomenon is revealed by the particular variation in the second-order autocorrelation function $g^{(2)}(\tau)=\langle I(t) I(t+\tau)\rangle_{t} /\langle I(t)\rangle_{t}\langle I(t+\tau)\rangle_{t}$ defined from the signal $I(t)$ emitted by the QS. ${ }^{15,16}$ For a single quantum system, $g^{(2)}(\tau)$ presents an antibunching dip when $\tau \rightarrow 0$. In a more general manner, $g^{(2)}(\tau)$ represents the joint probability of detecting a second photon at time $\tau$ knowing that a first one has been emitted at $\tau=0$, i.e., $\rho_{e e}(0)=0$. Consequently, the normalized second-order photon correlation function can be written as

$$
g^{(2)}(\tau)=\frac{\rho_{e e}(\tau)}{\rho_{e e}(\infty)},
$$

in which $\rho_{e e}(t)=\langle e|\rho(t)| e\rangle$ and $\rho_{e e}(\infty)$ is the stationary population of the excited state. The direct numerical resolution of the optical Bloch equations following from Eq. (4) gives access to the QS photon statistics for any illumination intensity. Nevertheless, let us note that for moderate laser intensity (weak Rabi frequency regime), we can assume that the coherences $\rho_{e g}(t)$ and $\rho_{g e}(t)$ adiabatically follow the population inversion $\eta(t)$. Within this approximation, the formal integration of the optical Bloch equations resulting from Eq. (4) is straightforward and the evolution of the system is governed by rate equations from which an analytical expression of the time-dependent excited state population $\rho_{e e}(t)$ can be obtained,

$$
\rho_{e e}(t)=\frac{\Delta\left(\mathbf{R}_{q s}\right) / 2}{\Delta\left(\mathbf{R}_{q s}\right)+\Gamma_{t o t}\left(\mathbf{R}_{q s}\right)}\left[1-e^{-\left(\Delta\left(\mathbf{R}_{q s}\right)+\Gamma_{t o t}\left(\mathbf{R}_{q s}\right)\right) t}\right],
$$

where $\Gamma_{t o t}\left(\mathbf{R}_{q s}\right)$ is the total decay rate of the excited level. This coefficient is proportional to the imaginary part of the surroundings field-susceptibility $\mathbf{S}\left(\mathbf{R}_{q s}, \mathbf{R}_{q s}, \omega_{e g}\right),{ }^{11}$

$$
\Gamma_{t o t}\left(\mathbf{R}_{q s}\right)=\frac{2 m_{e g}}{\hbar} \mathbf{u} \cdot \operatorname{Im}\left\{\mathbf{S}\left(\mathbf{R}_{q s}, \mathbf{R}_{q s}, \omega_{e g}\right)\right\} \cdot \mathbf{u} .
$$

In Eq. (6), $\Delta\left(\mathbf{R}_{q s}\right)$ represents the QS absorption rate,

$$
\Delta\left(\mathbf{R}_{q s}\right)=\frac{2 \Omega^{-} \Omega^{+} \Gamma_{t o t}\left(\mathbf{R}_{q s}\right)}{\delta_{L}^{2}+\Gamma_{t o t}^{2}\left(\mathbf{R}_{q s}\right) / 4}
$$

and

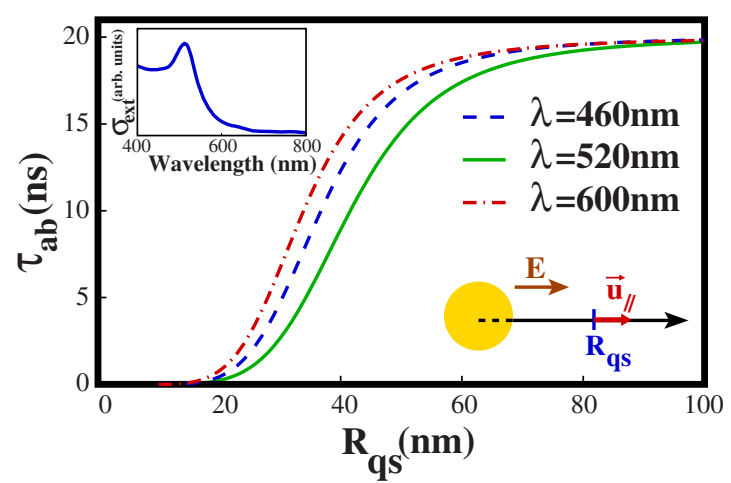

FIG. 2. (Color online) Antibunching time of a QS in the near field of a gold nanosphere in vacuum for a given orientation of the transition moment and three different wavelengths (particle diameter $D=20 \mathrm{~nm}) . \delta_{L}=0, \Gamma_{e}=1 / 20 \mathrm{~ns}^{-1}$, and vacuum Rabi frequency $\Omega_{0}=1 / 100 \mathrm{~ns}^{-1}=\Gamma_{e} / 5$. The inset represents the extinction spectra of the isolated gold sphere.

$$
\Omega^{-/+}=\sum_{\alpha=x, y, z} \frac{\Omega_{\alpha}\left(\mathbf{R}_{q s}\right)}{2} e^{(-/+) i \phi_{\alpha}\left(\mathbf{R}_{q s}\right)} .
$$

The second-order photon autocorrelation function $g^{(2)}(\tau)$ can be deduced from Eqs. (5) and (6),

$$
g^{(2)}(\tau)=1-e^{-\left[\Delta\left(\mathbf{R}_{q s}\right)+\Gamma_{t o t}\left(\mathbf{R}_{q s}\right)\right] \tau}
$$

from which an analytical expression of the antibunching time $\tau_{a b}$ can be defined,

$$
\tau_{a b}=\frac{1}{\Delta\left(\mathbf{R}_{q s}\right)+\Gamma_{t o t}\left(\mathbf{R}_{q s}\right)} .
$$

Relations (8) and (11) are interesting because they provide a simple expression of the antibunching time in which both total decay and absorption rates appear clearly. Nevertheless this assumption fails to properly describe the photon statistics for strong local-field-QS couplings. In this case, the autocorrelation function $g^{(2)}(\tau)$ exhibits Rabi oscillations ${ }^{17,18}$ that must be computed by direct time integration of Bloch equations [cf. curves of Fig. 4(f)]. In the following, three different applications illustrate the exposed framework in either the weak- or strong-coupling regime.

In a first step, we have investigated the evolution of the antibunching time $\tau_{a b}$ of a QS when entering the near field of an illuminated gold nanosphere. To perform the computation, the sphere has been discretized in volume using 1279 cells located at the nodes of a hexagonal compact array. ${ }^{19}$ Figure 2 shows the results for a fixed orientation $\mathbf{u}$ of the transition dipole moment and three different wavelengths. We have used the permittivity of gold $^{20}$ and the lifetime of the QS in vacuum is fixed at $1 / \Gamma_{e}=20 \mathrm{~ns}$. In that example, the vacuum Rabi frequency $\Omega_{0}$ remains small compared to the total decay rate so that the adiabatic approximation is still valid and expression (11) applies. Far from the gold nanosphere, the antibunching time $\tau_{a b}$ converges toward the lifetime of the excited state in vacuum. When entering the optical near field located around the particle, both excitation field and optical evanescent modes density increase. This effect is further enhanced when working near the plasmon resonance (solid 


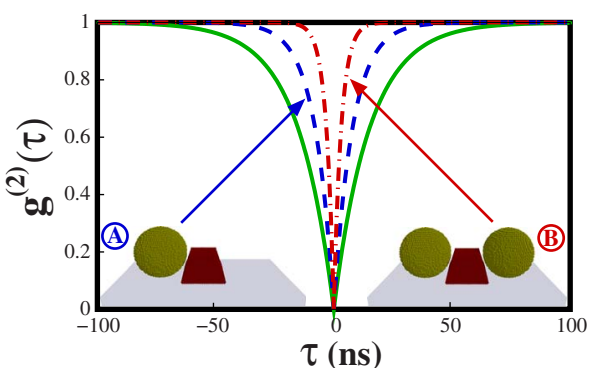

FIG. 3. (Color online) Second-order autocorrelation function of the photons emitted at $680 \mathrm{~nm}$ by a single NV center located in the center of a single nanodiamond. The nanodiamond $(\epsilon=6.0)$ is modeled by a truncated pyramid of square cross section (height $h=30 \mathrm{~nm}$, lower side $L=48 \mathrm{~nm}$, and upper side $l=38 \mathrm{~nm}$ ). The gold nanospheres have a diameter of $60 \mathrm{~nm}$ and are deposited on a glass substrate $(n=1.5)$. The dielectric constant has been taken from Ref. 20. $\delta_{L}=0, \Gamma_{e}=1 / 20 \mathrm{~ns}^{-1}$, and $\Omega_{0}=1 / 100 \mathrm{~ns}^{-1}=\Gamma_{e} / 5$. Solid line: result without gold nanosphere.

green curve: $520 \mathrm{~nm}$ ) of the spherical particle. At such weak Rabi frequency, the antibunching time $\tau_{a b}$ is mainly governed by the total decay rate which is composed of both radiative and nonradiative contributions. At very short distance from the particle surface, $\tau_{a b}$ decreases rapidly due to the nonradiative channels responsible for the metal quenching effect.

A closely related configuration recently studied in Schietinger et al. ${ }^{9}$ has been investigated in Fig. 3. It concerns the second-order autocorrelation function measurement of a single color center embedded in a nanodiamond when the hosting nanodiamond is surrounded by either a single gold particle (configuration A) or by two particles (configuration B). The parameters used for describing the NV center of diamond are summarized in the caption of Fig. 3. Two static configurations (A) and (B) are considered where gold particles and nanodiamond volumes are discretized with a hexagonal compact array. The NV emitter is located at the center of the nanodiamond. In good agreement with experimental data reported in Ref. 9, we observe a significant squeezing of the $g^{(2)}(\tau)$ dip. According to the result of Fig. 2, the observed reduction in $\tau_{a b}$ is a consequence of the significant amplification of $\Gamma_{t o t}\left(\mathbf{R}_{q s}\right)$ and of the optical near field generated by the gold particles. This is consistent with the results of Ref. 9 that demonstrated that passing from configuration (A), with a single gold sphere, to configuration (B), with two gold spheres, significantly increases the total radiative decay rate and the antibunching rate. Moreover, in the second configuration, the creation of hot spots between the two metal spheres enhances the excitation of the NV center via localfield effects. Our calculations are in excellent agreement with this experiment. They reproduce this result with an antibunching time $\tau_{a b}$ varying from $13.8 \mathrm{~ns}$ (configuration A) to 6.4 ns (configuration B).

Many other interesting behaviors of QS placed in the vicinity of complex near-field optical landscapes can be predicted with the model. In the application of Fig. 4, we have considered a second system of experimental interest ${ }^{10}$ in which a single NV center probes the optical near field generated by illuminating a glass sample supporting chromium

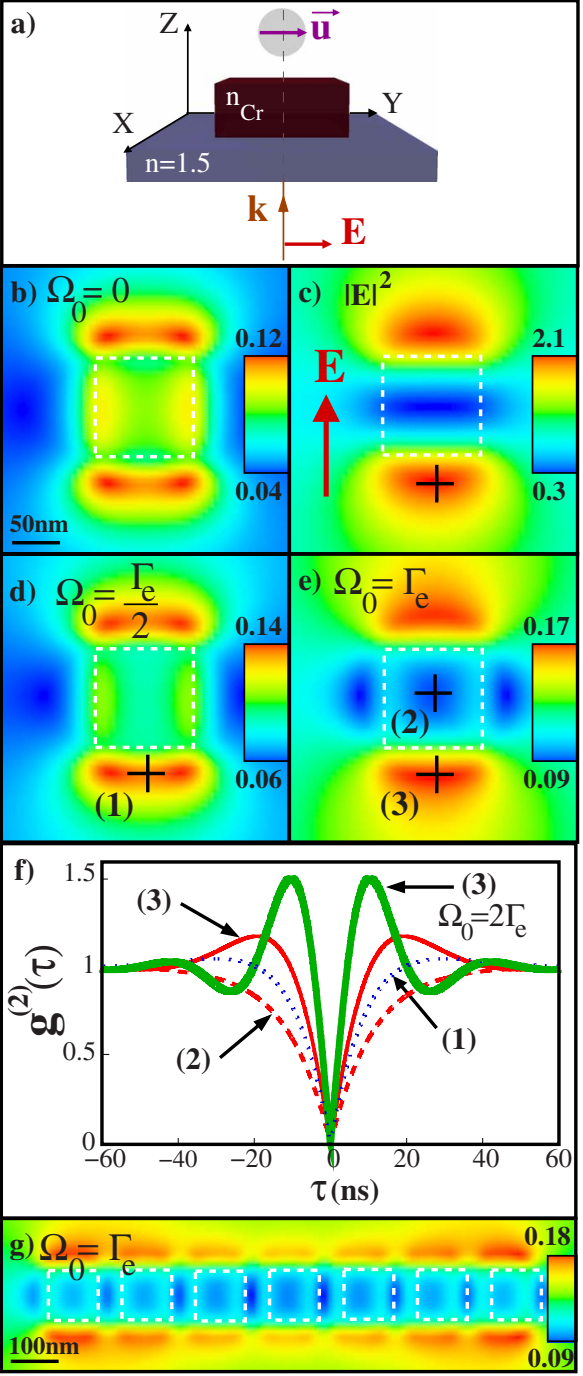

FIG. 4. (Color online) Photon statistics of a NV center $\left(\Gamma_{e}=1 / 20 \mathrm{~ns}^{-1}\right.$ ) located above chromium pads (height $=40 \mathrm{~nm}$, side $=100 \mathrm{~nm}$, center-to-center distance $=150 \mathrm{~nm}$ ). (a) Perspective view of a NV center scanning a single chromium pad illuminated with a normal incident plane wave. (b) Map of the total decay rate. (c) Field intensity map. [(d) and (e)] Evolution of the antibunching rate map $\left(\Gamma_{a b}=1 / \tau_{a b}\right)$ when passing from $\Omega_{0}=\Gamma_{e} / 2$ to $\Omega_{0}=\Gamma_{e}$. (f) Second-order correlation functions of a NV center at positions (1) (dotted line), (2) (dashed line), or (3) (solid line) of maps (d) and (e). Thick solid line: $\mathrm{NV}$ at position (3) and $\Omega_{0}=2 \Gamma_{e}$. (g) Antibunching rate map computed near a line of chromium pads $\left(\Omega_{0}=\Gamma_{e}\right)$.

microfabricated pads. We have computed the evolution of the antibunching rate $\Gamma_{a b}=1 / \tau_{a b}$ of the NV center when it is raster scanned across a plane parallel to the lithographically structured surface. The calculations presented in Figs. 4(b)-4(g) are performed for a transition dipole moment aligned along the (OY) axis and an incident electric field polarized along the same direction (normal incidence). The $\mathrm{NV}$ center is scanned at a constant distance $Z_{q s}=80 \mathrm{~nm}$ above the sample. The variations in the antibunching rate $\Gamma_{a b}\left(X_{q s}, Y_{q s}, Z_{q s}=80 \mathrm{~nm}\right)$ reveal a dramatic dependence of the photon statistics with respect to the lateral NV center $\left(X_{q s}, Y_{q s}\right)$ location: (i) when the laser intensity completely 
vanishes [see Fig. 4(b) where $\Omega_{0} \rightarrow 0$ ], the antibunching rate map corresponds to the total decay rate map which is generally proportional to the fluorescence intensity. In this limit case, according to relation (11), $\Delta\left(\mathbf{R}_{q s}\right)=0$ and $\Gamma_{a b}\left(\mathbf{R}_{q s}\right)=\Gamma_{t o t}\left(\mathbf{R}_{q s}\right)$. At this height $Z_{q s}=80 \mathrm{~nm}, \Gamma_{t o t}$ varies between $0.12 \mathrm{~ns}^{-1}$ (on the pad top) to $0.03 \mathrm{~ns}^{-1}$ (far from the pad top).

(ii) In the following maps (c)-(e), the laser illuminates the sample and produces the typical near field pattern represented in (c) where the intensity has been normalized with respect to the incident field intensity computed without any structure. When increasing the laser intensity [maps (d) and (e)], the photon bunching effect is reinforced along the pad sides and decreases on the top of the structures. A similar reversal contrast phenomenon was already observed in nearfield optics. ${ }^{21,22}$ As expected, a strong illumination regime is reached when $\Omega_{0}$ is comparable to $\Gamma_{e}$. In this regime [cf. Figs. 4(e) and 4(f)], a dramatic change in the second-order correlation function is observed when passing from a top site [labeled (2) in Figs. 4(e) and 4(f)] to the side site labeled (3) where we can observe the first modulations of the Rabi oscillation [see curve (3) of $g^{(2)}(\tau)$ ]. This change is induced by the strong variation in the optical near field between these two sites [see (c) map].

(iii) Finally, by keeping the illumination parameters of Fig. 4(e), we observe that an elongated antibunching area can be created when several dielectric pads are aligned [cf. map of Fig. 4(g)]. In other words, the optical near field produced by the pad chain tends to symmetrically produce areas with simultaneous photon antibunching and bunching zones that could be exploited for the emission control of single quantum systems. This calculation could also find a direct experimental counterpart in near-field optical microscopy using a nanodiamond-based single photon tip. ${ }^{10}$

In conclusion, we have developed a concise theoretical framework that can reproduce and analyze experimental photon statistics recorded on hybrid architectures composed of a quantum system coupled with complex dielectric or metallic nanostructures. By directly solving Bloch equations, all illumination regimes are accessible within the same numerical framework. The obtained equations provide information about the mechanisms responsible for the photon antibunching changes (spatial and polarization control, plasmon resonance effects,...). Our method enables direct comparison with the current experimental work and could be generalized without formal difficulties to more complex systems.

The authors would like to thank G. Dujardin, S. Huant, and A. Drezet for fruitful discussions. This work was supported by the computing facility center CALMIP of the University Paul Sabatier of Toulouse (France) and funded by the ANR Projects NAPHO and PlasTips.
${ }^{1}$ L. Novotny and B. Hecht, Principles of Nano-Optics, 1 st ed. (Cambridge University Press, London, 2006).

${ }^{2}$ S. A. Maier, Plasmonics: Fundamentals and Applications, $1 \mathrm{st}$ ed. (Springer, New York, 2007).

${ }^{3}$ A. V. Akimov, A. Mukherjee, C. L. Yu, D. E. Chang, A. S. Zibrov, P. R. Hemmer, H. Park, and M. D. Lukin, Nature (London) 450, 402 (2007).

${ }^{4}$ T. M. Babinec, B. J. M. Hausmann, M. Khan, Y. Zhang, J. R. Maze, P. R. Hemmer, and M. Loncar, Nat. Nanotechnol. 5, 195 (2010).

${ }^{5}$ H. G. Frey, S. Witt, K. Felderer, and R. Guckenberger, Phys. Rev. Lett. 93, 200801 (2004).

${ }^{6}$ P. Anger, P. Bharadwaj, and L. Novotny, Phys. Rev. Lett. 96, 113002 (2006).

${ }^{7}$ S. Kühn, U. Håkanson, L. Rogobete, and V. Sandoghdar, Phys. Rev. Lett. 97, 017402 (2006).

${ }^{8}$ Y. Sonnefraud, A. Cuche, O. Faklaris, J.-P. Boudou, T. Sauvage, J.-P. Roch, F. Treussart, and S. Huant, Opt. Lett. 33, 611 (2008).

${ }^{9}$ S. Schietinger, M. Barth, T. Aichele, and O. Benson, Nano Lett. 9, 1694 (2009).

${ }^{10}$ A. Cuche, A. Drezet, Y. Sonnefraud, O. Faklaris, F. Treussart, J. F. Roch, and S. Huant, Opt. Express 17, 19969 (2009).

${ }^{11}$ C. Girard, O. J. F. Martin, G. Lévèque, G. Colas des Francs, and
A. Dereux, Chem. Phys. Lett. 404, 44 (2005).

${ }^{12}$ C. Cohen-Tannoudji, J. Dupont-Roc, and G. Grynberg, Processus d'interaction entre photons et atomes (InterEditions, Paris, 1988).

${ }^{13}$ G. Colas des Francs, C. Girard, J. C. Weeber, C. Chicane, T. David, A. Dereux, and D. Peyrade, Phys. Rev. Lett. 86, 4950 (2001).

${ }^{14}$ G. Baffou, C. Girard, E. Dujardin, G. Colas des Francs, and O. J. F. Martin, Phys. Rev. B 77, 121101(R) (2008).

${ }^{15}$ M. Orrit, Single Mol. 3, 255 (2002).

${ }^{16}$ Y. Zheng and F. L. H. Brown, Phys. Rev. Lett. 90, 238305 (2003).

${ }^{17}$ G. Wrigge, I. Gerhardt, J. Hwang, G. Zumofen, and V. Sandoghdar, Nat. Phys. 4, 60 (2008).

${ }^{18}$ Y. Gu, L. Huang, O. J. F. Martin, and Q. Gong, Phys. Rev. B 81, 193103 (2010).

${ }^{19}$ C. Girard, E. Dujardin, G. Baffou, and R. Quidant, New J. Phys. 10, 105016 (2008).

${ }^{20}$ P. B. Johnson and R. W. Christy, Phys. Rev. B 6, 4370 (1972).

${ }^{21}$ J. C. Weeber, E. Bourillot, A. Dereux, J. P. Goudonnet, Y. Chen, and C. Girard, Phys. Rev. Lett. 77, 5332 (1996).

${ }^{22}$ D. Barchiesi, C. Girard, O. J. F. Martin, D. Van Labeke, and D. Courjon, Phys. Rev. E 54, 4285 (1996). 